\title{
Expanding Treatment Options for Metastatic Prostate Cancer
}

\author{
Emmanuel S. Antonarakis, M.D. and Mario A. Eisenberger, M.D. \\ Johns Hopkins Sidney Kimmel Comprehensive Cancer Center, Baltimore
}

Prostate cancer is an androgen-receptor-dependent disease, and the blocking of androgenreceptor signaling is a hallmark of prostate-cancer therapeutics. Although most patients initially benefit from therapy involving deprivation of gonadal androgen, the disease eventually progresses after 12 to 48 months, depending on the disease burden, host factors, and inherent tumor biology. After progression, the disease evolves into a new clinically and molecularly heterogeneous state known as castration-resistant prostate cancer, which is invariably fatal.

The clinical course of metastatic castration-resistant prostate cancer has changed considerably, primarily because of factors such as earlier diagnosis, stage migration, and changes in practice patterns. Earlier initiation of androgen-deprivation therapy and the increased use of diagnostic imaging have contributed to earlier detection of metastatic disease in androgen-deprived patients. ${ }^{1}$ Furthermore, new treatments have further extended the time to the terminal phase of the disease. Although precise figures are not available, the duration of the course of metastatic castration-resistant prostate cancer measured from the first documented metastasis (in the castrate state) until death may now extend beyond 5 years (Fig. 1).

Androgen-receptor signaling remains active even with castrate levels of serum testosterone ( $<50 \mathrm{ng}$ per deciliter), contrary to the previous notion that disease progression after gonadal ablation necessarily implied androgen-independent escape mechanisms. Restoration of ligand-dependent androgen-receptor signaling after castration has been documented and offers new opportunities for targeted approaches to treatment, many of which are currently in clinical development. ${ }^{2}$ One way in which castration-resistant prostate cancer maintains androgen-receptor signaling is by overexpressing cytochrome P-450c17 (CYP17), a key enzyme in extragonadal (adrenal, prostatic, and intratumoral) androgen biosynthesis.

In this issue of the Journal, de Bono and colleagues ${ }^{3}$ report the results of a multicenter phase 3 trial evaluating the oral CYP17 inhibitor abiraterone acetate (administered with prednisone) as compared with placebo and prednisone in men with metastatic castrationresistant prostate cancer who had disease progression after docetaxel chemotherapy. The trial showed that abiraterone plus prednisone was safe and associated with a 35\% reduction in the risk of death as compared with placebo plus prednisone, with a median survival of 14.8 months among patients who received abiraterone acetate plus prednisone versus 10.9 months among patients who received placebo plus prednisone. In addition, abiraterone treatment resulted in an improved prostate-specific antigen (PSA) response rate, an improved objective response rate on the basis of radiographic findings, longer time to disease progression on the basis of the PSA level, and longer progression-free survival on the basis of radiographic findings.

Copyright () 2011 Massachusetts Medical Society. All rights reserved.

Disclosure forms provided by the authors are available with the full text of this article at NEJM.org. 
Given our understanding of abiraterone's mechanism of action, the findings provide a proof of principle that metastatic castration-resistant prostate cancer remains androgen-driven. This notion has previously been suggested based on the modest efficacy observed with the use of high-dose ketoconazole, ${ }^{4}$ a nonselective competitive inhibitor of CYP17; however, that agent has more toxicity than abiraterone. In addition, although this study led to the approval of abiraterone by the Food and Drug Administration (FDA) on April 28, 2011, for patients who had received docetaxel, these results provide sufficient evidence of efficacy to justify the use of abiraterone in all patients with metastatic castration-resistant prostate cancer (i.e., even those with no previous chemotherapy treatment). The role of abiraterone in the treatment of prostate cancer is also being examined in an ongoing placebo-controlled, phase 3 trial involving patients with metastatic castration-resistant prostate cancer who had not received chemotherapy and in studies involving patients with earlier stages of the disease.

Until recently, life-prolonging therapies for patients with metastatic castration-resistant prostate cancer were limited and consisted only of docetaxel ${ }^{5}$ (approved by the FDA in 2004 as first-line chemotherapy for metastatic castration-resistant prostate cancer), whereas mitoxantrone had previously been approved based on improvements in quality of life. ${ }^{6}$ In 2010, two additional therapies became FDA-approved treatment options for men with metastatic castration-resistant prostate cancer: the autologous immunotherapy product sipuleucel- $\mathrm{T}^{7}$ (indicated for men with minimal or no symptoms) and the chemotherapy agent cabazitaxel $^{8}$ (indicated for men with disease progression after docetaxel treatment). Also in 2010, after showing superiority against the previously approved bisphosphonate zoledronic acid, the bone-targeting agent denosumab was FDA- approved for the prevention of skeletalrelated events in men with metastatic castration-resistant prostate cancer. ${ }^{9}$

Although treatment choices for men with metastatic castration-resistant prostate cancer have increased and outcomes have improved (Fig. 1), the duration of progression-free and overall survival still remains relatively short. It is clear, however, that our knowledge of the biologic mechanisms involved in the progression of metastatic castration-resistant prostate cancer has reached a level at which the discovery of more effective targeted approaches will probably further improve outcomes. Data are lacking to define the optimal sequence and combination of abiraterone with other agents, to determine the activity and toxicity of other androgenreceptor-targeting drugs (e.g., MDV3100) or compounds that may destabilize androgenreceptor variants encoded by spliced transcripts lacking the androgen-binding domain but remaining constitutively active, ${ }^{10}$ and to integrate androgen-receptor-directed therapies with other therapies.

Is it still realistic to demand that new agents increase overall survival among patients with metastatic castration-resistant prostate cancer? Survival is likely to become harder to assess, unless we are able to control for the influence of subsequent treatments on survival. More recent trials in metastatic castration-resistant prostate cancer have used coprimary end points such as progression-free survival and overall survival in an attempt to validate progressionfree survival as a clinically meaningful and more feasible end point. Furthermore, clinical, radiologic, biologic, and genomic response biomarkers should be identified and validated; these may act as surrogates of clinical benefit and may help in the selection of appropriate patients for specific therapies. In addition, the approach of regulatory agencies to consider patients who have received chemotherapy as a distinct group from those who have not may need to be rethought. Currently, a trial leading to approval of a drug for patients who have previously received chemotherapy may not lead to approval of the same drug for patients who have not received chemotherapy unless a second clinical trial involving the latter group is conducted. It is not clear that a second trial should be required. Finally, placebo-controlled 
trials may no longer be ethical or feasible in men with metastatic castration-resistant prostate cancer.

\section{References}

1. Cooperberg MR, Lubeck DP, Meng MV, Mehta SS, Carroll PR. The changing face of low-risk prostate cancer: trends in clinical presentation and primary management. J Clin Oncol. 2004; 22:2141-9. [PubMed: 15169800]

2. Knudsen KE, Scher HI. Starving the addiction: new opportunities for durable suppression of AR signaling in prostate cancer. Clin Cancer Res. 2009; 15:4792-8. [PubMed: 19638458]

3. de Bono JS, Logothetis CJ, Molina A, et al. Abiraterone and increased survival in metastatic prostate cancer. N Engl J Med. 2011; 364:1995-2005. [PubMed: 21612468]

4. Small EJ, Halabi S, Dawson NA, et al. Antiandrogen withdrawal alone or in combination with ketoconazole in androgen-independent prostate cancer patients: a phase III trial (CALGB 9583). J Clin Oncol. 2004; 22:1025-33. [PubMed: 15020604]

5. Tannock IF, de Wit R, Berry WR, et al. Docetaxel plus prednisone or mitoxantrone plus prednisone for advanced prostate cancer. N Engl J Med. 2004; 351:1502-12. [PubMed: 15470213]

6. Tannock IF, Osoba D, Stockler MR, et al. Chemotherapy with mitoxantrone plus prednisone or prednisone alone for symptomatic hormone-resistant prostate cancer: a Canadian randomized trial with palliative end points. J Clin Oncol. 1996; 14:1756-64. [PubMed: 8656243]

7. Kantoff PW, Higano CS, Shore ND, et al. Sipuleucel-T immunotherapy for castration-resistant prostate cancer. N Engl J Med. 2010; 363:411-22. [PubMed: 20818862]

8. de Bono JS, Oudard S, Ozguroglu M, et al. Prednisone plus cabazitaxel or mitoxantrone for metastatic castration-resistant prostate cancer progressing after docetaxel treatment: a randomised open-label trial. Lancet. 2010; 376:1147-54. [PubMed: 20888992]

9. Fizazi K, Carducci M, Smith M, et al. Denosumab versus zoledronic acid for treatment of bone metastases in men with castration-resistant prostate cancer: a randomised, double-blind study. Lancet. 2011; 377:813-22. [PubMed: 21353695]

10. Hu R, Dunn TA, Wei S, et al. Ligand-independent androgen receptor variants derived from splicing of cryptic exons signify hormone-refractory prostate cancer. Cancer Res. 2009; 69:16-22. [PubMed: 19117982] 


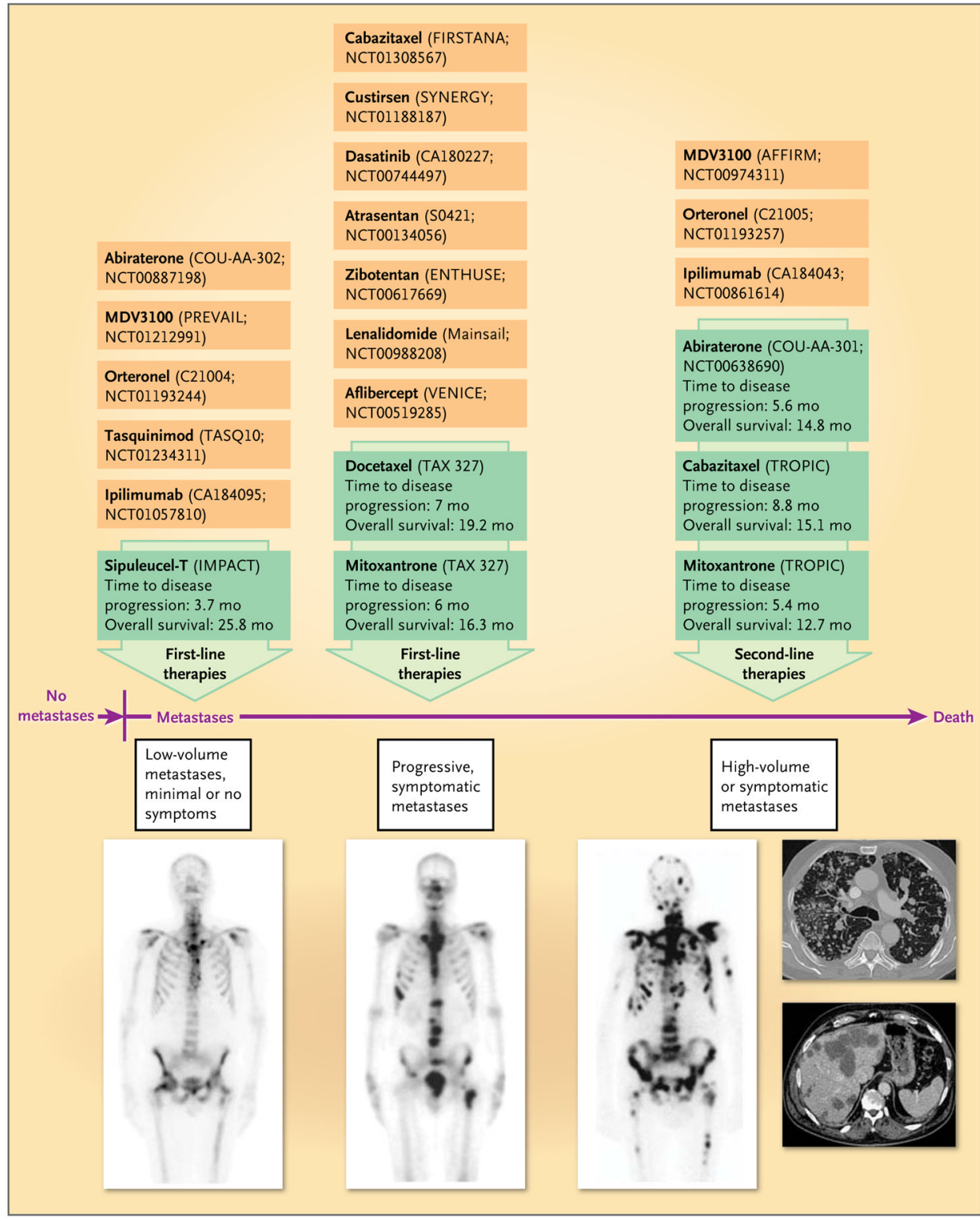

Figure 1. Evidence-Based Therapies for Metastatic Castration-Resistant Prostate Cancer, and Selected Emerging Treatments

Radionuclide bone scans show the typical progression of metastatic castration-resistant prostate cancer from limited bone involvement (left) to progressive bone metastases (middle) to extensive metastases (right). The computed tomographic scans on the far right show visceral (lung and liver) metastases. Green boxes indicate agents that are currently approved by the Food and Drug Administration for the treatment of metastatic castrationresistant prostate cancer. The effects of these agents on disease progression and overall survival are shown. Orange boxes indicate selected agents currently in phase 3 drug development. 